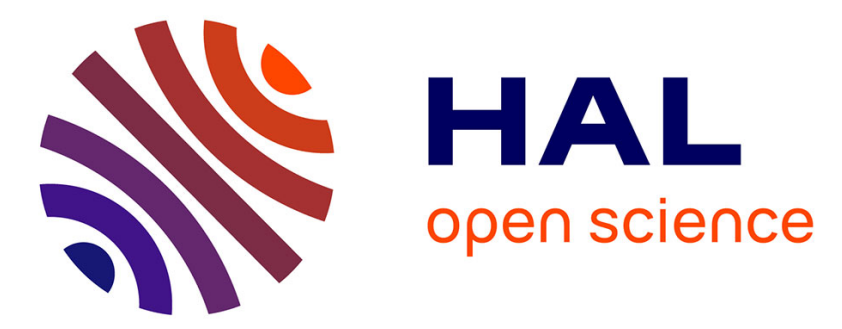

\title{
Potentiality in Physics
}

\author{
Max Kistler
}

\section{To cite this version:}

Max Kistler. Potentiality in Physics. Handbook of Potentiality, Springer Netherlands, pp.353 - 374, 2018, 10.1007/978-94-024-1287-1_14 . hal-02565369

\section{HAL Id: hal-02565369 \\ https://hal.science/hal-02565369}

Submitted on 6 May 2020

HAL is a multi-disciplinary open access archive for the deposit and dissemination of scientific research documents, whether they are published or not. The documents may come from teaching and research institutions in France or abroad, or from public or private research centers.
L'archive ouverte pluridisciplinaire HAL, est destinée au dépôt et à la diffusion de documents scientifiques de niveau recherche, publiés ou non, émanant des établissements d'enseignement et de recherche français ou étrangers, des laboratoires publics ou privés. 


\title{
Potentiality in Physics \\ Max Kistler
}

in Kristina Engelhard \& Michael Quante (eds.), Handbook of Potentiality, Dordrecht: Springer, 2018, p. 353-374.

DOI: 10.1007/978-94-024-1287-1_14

Université Paris 1 Panthéon-Sorbonne, CNRS, ENS

UMR 8590 IHPST - Institut d'Histoire et de Philosophie des Sciences et des Techniques mkistler@univ-paris1.fr

\begin{abstract}
The term "potentiality" is nowadays used neither in physics nor in most philosophical interpretations of physical theories. However, it can be helpful to use it in the context of the metaphysical interpretation of both classical and quantum physics. Potentiality is only pragmatically different from dispositionality. The use of the term "potentiality" is appropriate under two conditions: 1) the actualization takes (more or less) time and 2) the potentiality makes its actualization (more or less) probable but not necessary. Examples in classical physics are potentials (such as the gravitational potential) and capacities (such as heat capacity). Heisenberg used the concept of potentiality in his interpretation of quantum mechanical systems in superposed states. I develop an interpretation of Heisenberg's conception according to which, 1) a quantum system that is, with respect to a given observable, in a superposed state, is potentially (but not actually) in one of its available eigenstates; 2) the superposed state itself is a powerful state that plays the role of a theoretical property: It provides a unifying explanation of a set of dispositions, corresponding to the measurement of various observables, where the measurement is the triggering condition and the outcome the manifestation.
\end{abstract}

\section{Introduction}

"Potential" is a technical term in physics, "potentiality" is not. Potentiality is a general philosophical concept that can also be applied to physical phenomena. In this chapter, I will concentrate on the concept of potentiality. However, before I ask how the concept of potentiality is or can be used in a philosophical interpretation of physics, I begin with a brief presentation of the physical concept of potential.

Potentials can be used to represent physical systems in which all forces are conservative $^{1}$. In that case, one can represent the system by the potential $\Psi$, which is a simple mathematical function of spatial location only: contrary to a vector, which has several components, the potential is a scalar quantity, which has only one component. The potential $\Psi$ can be used to retrieve various other physical quantities. Let us consider a system in which only gravitational forces are acting. The potential energy $\mathrm{U}(\mathrm{x})$ of an object with mass $\mathrm{m}$, situated at a given point $\mathrm{x}$ in space, is the product of $\mathrm{m}$ with the value of the potential $\Psi(\mathrm{x})$ at point $\mathrm{x}$.

$$
U=m \Psi .
$$

\footnotetext{
${ }^{1}$ All fundamental forces are conservative. Non-conservative forces, such as friction, only appear in incomplete representations of physical systems. See Feynman et al. (1963, p. 14-6/7). The presentation of the relations between the different physical concepts in this paragraph follows Feynman et al.'s (1963) exposition.
} 
The force $\mathrm{F}$ acting on the object in a given direction $\mathrm{dx}$ is the partial derivative of $\mathrm{U}$ in the direction $\mathrm{dx}$.

$$
F_{x}=-\partial U / \partial x^{2}
$$

(The force as a vector in three dimensions can be written $F=-\nabla U$, where " $\nabla$ " represents the gradient operator).

The force can be considered as a power to accelerate objects that undergo it. From the potential, one can also calculate, at each point of space, the gravitational field $\mathrm{C}$, which is the force acting on a body located at that point, per unit of mass: $\mathrm{C}=\mathrm{F} / \mathrm{m}$.

$$
C=-\nabla \Psi=-\nabla U / m=F / m \text {. }
$$

Equivalently, the potential energy of a body with mass $m$ at a given point $x_{0}$ is equal to the work $^{3}$ required to bring the body to that point, from infinitely far away, where the strength of gravitational forces is taken to be zero, and where the potential energy is by convention taken to be zero.

$$
U\left(x_{0}\right)=-\int_{\infty}^{x_{0}} F d x
$$

In the same way, the (gravitational) potential of the system at point $\mathrm{x}_{0}$ is equal to the work per mass required to bring a massive body to that point:

$$
\Psi\left(x_{0}\right)=-\int_{\infty}^{x_{0}}(F / m) d x=-\int_{\infty}^{x_{0}} C d x
$$

As I have just presented its definition and use, the gravitational potential appears as a mathematical construction. Only the field and forces are real and causally efficacious, whereas the gravitational potential is nothing more than a mathematically convenient way of representing the gravitational field and forces. This is indeed how potentials are often interpreted. However, the interpretation of potentials as mathematical fictions does not seem to be adequate for all potentials. The electromagnetic potential can be defined in a similar way to the gravitational potential. As the gravitational potential, it appears to be a mathematical device from which the physically real electromagnetic field and forces may be derived. However, the electrodynamic potential may exercise causal influence at points at which the field strengths are zero ${ }^{5}$. According to the Causal Criterion of Reality ${ }^{6}$, this is a good reason to regard the potential as real in itself, over and above the field and forces that can be derived from it.

\footnotetext{
${ }^{2}$ Here is a way to understand the minus sign. The gravitational potential around a massive body $\mathrm{M}$ is by convention set to be zero at infinite distance from the body. The value of the potential is increasingly negative with decreasing distance from $\mathrm{M}$. The derivate of the potential with respect to positive distance from $\mathrm{M}$ is positive, but the gravitational force is directed in the opposite direction, i.e. towards $M$.

${ }^{3}$ Work, a form of energy, is defined as the integral taken over the path of the body, of the force acting on the body at each point on the path.

${ }^{4}$ To understand the minus sign: Positive work (energy) is required to remove a body from the proximity of the source of the potential, i.e. the work is positive if the direction of the movement is directed against the direction of the force.

5 This is the Aharonov-Bohm effect. Cf. Healey (1997), Belot (1998).

6 According to Causal Criterion of Reality, all and only those entities exist that make a causal difference. Armstrong has called it the "Eleatic principle" by reference to its formulation by the Eleatic Stranger in Plato's Sophist (247d-e). In Armstrong's words, "everything that exists makes a difference to the causal powers of something" (Armstrong 1997, p. 41), but it is the converse that is used to justify the postulation of the existence of both particulars and properties: Everything that makes a difference to the causal powers of something exists. The justification of the principle itself rests on the claim that it is a central part of scientific methodology. Jaegwon Kim called it "Alexander's Dictum" (Kim 1992, p. 134), in honour of Samuel Alexander (1920) who defended it as a metaphysical principle: "To be real is to have causal powers" (Kim 1992, p. 135; Kim's italics). Cf. Kistler (2002).
} 
The concept of potentiality, though the word is not used within physics, played a role in the philosophical interpretation of superposed states of quantum mechanical systems. Heisenberg (1958, p. 27) suggested that the wave function describing the state of certain quantum systems - those in so-called superposed states ${ }^{7}$ - describes potentialities of the system, in a sense in which potentialities are not actual properties. ${ }^{8}$ In this context, Heisenberg uses the term "potentia" (Heisenberg 1958, p. 15, 154, 155) that belongs to the Aristotelian tradition.

The use of the concepts of potentiality and actuality in the interpretation of quantum mechanics is sometimes accompanied by the thesis that only superposed states of quantum systems are potentialities, but not the states of "classical" physical systems. Some authors who follow Heisenberg in interpreting quantum states as potentialities, judge that "a substantial distinction between potential and actual existence is rendered obsolete in classical mechanics" (Karakostas 2007, p. 281). However, Aristotle introduced the concepts of potentiality and actuality to make sense of change in general. Thus in order to defend the claim $^{9}$ that only certain quantum mechanical systems have (or are) potentialities, whereas physical objects that can be described in the terms of classical physics do not, the concepts of potentiality and actuality need to be given a new and more specific sense.

This chapter will have two parts. In the first part, I present a general interpretation of the conceptual role of potentiality in physics, based on a general thesis on the relation between the concepts of potentiality and dispositionality. In the second part, I will suggest a realist interpretation of quantum mechanical systems in terms of powers and dispositions, which I will develop by starting from a critical analysis of Heisenberg's thesis that quantum mechanical systems in superposed systems have only potential, but no actual, existence. I will suggest that superposed quantum states are theoretical properties that can be conceived as powers, which are indirectly characterized by a specific set of dispositions.

\section{Potentiality as dispositionality}

I this first part of the chapter, I argue that the terms "potentiality" and "dispositionality" have the same meaning. A potentiality is a disposition, in the sense this concept has in metaphysics and philosophy of language. The difference between the terms "potential" and "dispositional" is, I would suggest, pragmatic.

The words "disposition" and "dispositional", as well as similar terms, such as "potency" (Bird 2007) are philosophical terms of art and not technical terms of physics. Other terms, such as "capacity" (Cartwright 1989) are technical terms of physics but are also used as philosophical concepts whose domain of application exceeds that of the homonymous technical term. The terms "potential" and "capacity" are used in physics with a meaning that is very similar to that of the philosophical concepts of potentiality and dispositionality. These concepts are the object of an amount of important literature in metaphysics, philosophy of language and philosophy of science. The names of some physical entities, such as "gravitational potential" and "heat capacity", indicate that they are potentialities or capacities. However, the philosophical concepts of potentiality and disposition also apply more generally to physical entities or properties whose potentiality or dispositionality is not reflected in their name. Such entities or properties are neither called "potentials" nor "capacities" nor

\footnotetext{
${ }^{7}$ I will explain shortly what superposed states are.

${ }^{8}$ The fact that potentialities, as Heisenberg uses the term, are non-actual properties of physical systems shows that they are very different from potentials, as the term is used in physics and briefly explained above. Potentials are actual and underlie actual fields and forces acting on objects.

${ }^{9}$ As we will see in the second part of the chapter, this claim, explicitly made by Karakostas (2007), is part of Heisenberg's conception of the difference between quantum mechanical systems and classical systems.
} 
"dispositions" nor do their names have a suffix like "-ible", "-able", "-uble", "-ability", ubility" etc. However, they share the characteristics of potentiality and dispositionality with entities that are explicitly called "potentials" or "capacities", i.e. they are both concepts that characterize entities indirectly, through the manifestations they cause in certain situations, which are called their "triggering" conditions.

It is essential to both concepts (dispositionality and potentiality) that any property to which one of them applies is essentially linked to a second property: for a disposition, the second property is its manifestations, for a potentiality, it is its actualization. I suggest that, although the meaning of these concepts is the same, there is a pragmatic difference between their uses, and this is in particular the case with their use in the interpretation of physics. Before I explain this difference, let me first introduce the philosophical concept of a disposition (See Mumford (1998), Kistler \& Gnassounou (2007)).

There are many common sense dispositions. Window panes are solid, transparent, and fragile, rubber is elastic. Dispositions are not directly observable, but there is usually a quite direct link between them and observable properties, which are their manifestations. The link can only be expressed by counterfactual conditionals. Such "counterfactuals", as they are also called, link a disposition with one or several pairs of conditions that are called, respectively, the disposition's "triggering" and "manifestation" conditions. To say that a window pane is fragile implies that, if it were strongly struck in normal or ordinary circumstances it would break. One can express this by saying that the truth condition of the proposition that window pane $\mathrm{w}$ is fragile is the same as the truth condition of the counterfactual proposition that if $\mathrm{w}$ was struck strongly in normal circumstances, w would break. It is necessary to include a clause like "in normal circumstances", often called a "ceteris paribus" clause, because the equivalence does not hold without exceptions. Typically, dispositions have what has been metaphorically labelled "antidotes" (Bird (1998)). To be toxic is a dispositional property: a substance $t$ is toxic for a given organism if it is the case that if the organism came, in normal circumstances, into contact with $\mathrm{t}$ (by ingestion, inhalation, touch etc.), it would be harmed or die. However, the counterfactual analysis of the meaning of the word "toxic" would be incorrect without the qualifier "in normal circumstances". Indeed, organisms can often be immunized against toxic substances by antidotes. An antidote for a given toxic substance $t$ is a substance such that, if an organism has absorbed this antidote for $t$, then it is not the case that if the organism came into contact with $t$ (and if circumstances were otherwise normal) it would be harmed.

Some dispositions manifest themselves independently of any triggering conditions (Mumford and Anjum (2011, p. 36)). Gravitation and radioactivity are cases in point. Massive objects have the disposition to be attracted by other massive objects, and this disposition manifests itself (indirectly) without any triggering. It contributes to the net force acting on the object, which manifests itself in the object's acceleration. Radioactive decay too needs no triggering.

The question of what makes true the attribution of a disposition is controversial. One thesis is that what makes true the attribution of a disposition is always a "categorical" property ${ }^{10}$. It is difficult to define the concept of a categorical property otherwise than by opposing it to that of a dispositional property, but categorical properties are usually taken to be structural, and typically microscopic, properties. The categorical properties that are taken to provide the truth-maker for ascriptions of fragility are micro-structural properties, such as properties of the chemical bonds between the molecules of the fragile object. According to the main alternative thesis, dispositional properties, in this context often called "powers", are properties in their own right, which are real and causally efficacious just as "categorical" or

10 Quine (1966, 73), Quine (1971, 10), Prior, Pargetter, Jackson (1982), Armstrong (1973), Armstrong (1997). 
structural properties. The relation between a macroscopic disposition such as fragility and the microscopic structural and chemical properties underlying it is reduction: the microscopic structure may reductively explain the fragility of the macroscopic window pane. However, the possibility of micro-reduction is independent from the issue whether a given property is dispositional or categorical. The microscopic properties in the reduction base may themselves be dispositional. Indeed, micro-reducibility does not seem to be necessary for being a disposition because at least some (maybe all) fundamental (i.e. non-reducible) properties, such as mass and charge, are dispositiona $1^{11}$. It does not seem to be sufficient either, for some macroscopic categorical properties, such as structural properties, are reducible: the cubic structure of a crystal is reducible in geometric terms to the geometrical relations between its atomic or molecular components.

All this applies to physical dispositions such as electric conductivity or heat capacity. Electrical conductivity (usually represented by the symbol $\sigma$ ) is a property of metals (and other materials), which is defined by the fact that if an object is electrically conductive, then if the object is put in an electric field of strength $\mathrm{E}$ then electric current of density $\mathrm{J}=\sigma \mathrm{E}$ will flow through $\mathrm{it}^{12}$. Placing the object in the electric field is the triggering condition of the disposition and the current flow its manifestation. Physics is full of such dispositional properties. As other dispositions, potentials and capacities in the technical sense of physics can be characterized by specific pairs of triggering conditions and manifestations. Saying that something has a potentiality (or a potential) for some effect means that it has a disposition for that effect.

Even though potentials are dispositions and everything that has potentiality also has dispositionality, it does not always seem appropriate to attribute potentiality to dispositional entities or properties. It may be just a contingent fact about language that some dispositional physical properties are called "potentials" or "capacities", whereas others, such as electric conductivity, are not. But "potentiality" is a philosophical term, which doesn't appear in physics texts any more than the terms "disposition" and "dispositionality". So it is a philosophical issue whether the concepts of dispositionality and potentiality are equivalent. My suggestion is that they share their literal meaning but not the pragmatic conditions of their appropriate use.

The first pragmatic constraint that distinguishes the use of the terms "potentiality" and "dispositionality" concerns the time at which a disposition manifests itself and the time at which a potentiality is actualized. The difference can be expressed in terms of the possibility of simultaneous attribution: it can be appropriate to attribute a disposition to something at a time at which the disposition manifests itself, but it seems less appropriate to attribute a potentiality at a time at which it is actualized.

Let us suppose that a system $\mathrm{s}$ has at time $\mathrm{t}$ a dispositional property $\mathrm{P}$. This means that if $\mathrm{s}$ were subject to the triggering condition (or one of the set of triggering conditions if there is more than one) $\mathrm{T}$ at time $\mathrm{t}$, it would manifest $\mathrm{M}$ at time $\mathrm{t}^{*}$ ( $\mathrm{t}^{*}$ may be equal to $\mathrm{t}$ or later than t). Take $\sigma$ to be electrical conductivity and $w$ to be a copper wire that has $\sigma$. Then for all times $\mathrm{t}$, if $\mathrm{w}$ is at $\mathrm{t}$ in an electric field $\mathrm{E}$, it will manifest electric current with current density $\mathrm{J}=\sigma \mathrm{E}$ at $\mathrm{t}^{13}$

11 On fundamental, irreducible or "ungrounded" dispositions, see Blackburn (1990), Molnar (2003), McKitrick (2003), Mumford (2006), Kistler (2007).

${ }^{12}$ The current density $\mathrm{J}$ is defined as the current per unit cross-sectional area of a conductor. For a given field E, the electric current I flowing through a wire depends on E, the wire's conductivity $\sigma$ and on the wire's crosssection.

13 In case the disposition is attributed at $t$ but triggered only at $t^{*}$ (where $t^{*}$ is later than $t$ ), it must be presupposed that the dispositional property $\mathrm{P}$ is a permanent property of the system, which persists from $t$ to $t^{*}$, 
It seems perfectly appropriate to attribute the disposition to $\mathrm{w}$ at time $\mathrm{t}$, at which it is triggered, even if it manifests itself at the same moment $t$ by the flow of current. However, it is central to the concept of a disposition that it can also be attributed at times $t$ at which it is not triggered. Therefore, a wire can correctly be said to be conductive during periods in which it is not subject to the triggering condition, even if it is never put in any electric field, and therefore never manifests its conductivity ${ }^{14}$.

By contrast, and this is the first aspect by which the use of the term "potentiality" differs from the use of the term "disposition", it is less appropriate to attribute a system s at time $t$ with the potentiality $\mathrm{P}$ for some actualization A if P's actualization A has already occurred at $t$. (The actualization of a potentiality is analogous to the manifestation of a disposition.) A property is more naturally considered to be a potentiality if the manifestation is expected to occur after some finite delay, relative to the ascription of the potentiality ${ }^{15}$. There is no such constraint for the use of "disposition". It can be appropriate to attribute a disposition at a time at which it is manifested.

There seems to be no difference with respect to the need for triggering: just as dispositions, some potentials but not all need triggering to be actualized. Just as dispositions, those potentials that require triggering for their actualization can exist untriggered.

Take a simple pendulum. When the mass of the pendulum starts its movement at a given height $h$, its potential energy is a function of $h$. On its downward movement, this potential energy is gradually transformed into kinetic energy, which is its actualization. When it reaches the lowest point on its trajectory, the potential energy has become completely actualized and entirely been transformed in kinetic energy. At least a part of the potential energy that the pendulum's mass has when it is above its lowest position, satisfies the condition of appropriateness for potentiality: it is not yet actualized and will only be actualised after some finite interval of time. Another example for potentiality in physics is radioactive decay. There is a delay between the truthful attribution of the potentiality for decay to a nucleus and its actualization. Each type of radioactive nucleus has a characteristic half-life, which is necessarily greater than zero. ${ }^{16}$ Thus, the actualization condition, which consists in the presence of the decay products, is realized later than the moment at which the potentiality for decaying is attributed to the nucleus. The potentiality of radioactive decay is incompatible with its actualization. Once the decay has occurred, the radioactive nucleus that

so that the system still possesses $\mathrm{P}$ at the time $\mathrm{t}^{*}$ of triggering. This requirement is explicitly expressed in Lewis (1997), one of the most recent and sophisticated analyses of dispositions in terms of counterfactual conditionals.

14 The question of whether it is appropriate to attribute a disposition at a time at which it manifests itself must be distinguished from the question of whether there is a delay between the triggering and the manifestation of a disposition. The concept of a disposition neither requires a delay between triggering and manifestation nor the absence of such a delay. Both delayed and immediate manifestations are conceivable and compatible with dispositionality. In the case of dispositions, there is room for variation along three dimensions. First, a system having disposition $\mathrm{P}$ at $\mathrm{t}$ may be triggered at any time, at $\mathrm{t}$ or later. Second, if the disposition is triggered at $\mathrm{t}$, no conceptual restriction is imposed on the time at which the characteristic manifestation appears: It may be simultaneous to triggering or follow triggering after a certain delay. Third, some dispositions need no triggering at all but always manifest, so to say, spontaneously.

${ }^{15}$ What is crucial here is the time at which the potential is attributed or ascribed. This is to be distinguished from the question when the object has acquired the potential. The latter question is independent of the distinction between potentiality and dispositionality that is the target of the present analysis. Say the radioactive nucleus $n$ decays at time $t_{1}$. At some earlier time $t_{0}$, when it has not yet decayed, we may truthfully attribute to $n$ the potentiality of decaying. The truth of the attribution of this potentiality to $n$ at time $t_{0}$ is completely independent of the time at which $\mathrm{n}$ has acquired that potentiality, which is the time at which it came into existence.

${ }_{16}$ An atomic nucleus of Plutonium $240\left({ }^{240} \mathrm{Pu}\right)$, e.g., has at time the potential to be transformed by radioactive decay into a nucleus of Uranium $236\left({ }^{236} \mathrm{U}\right)$. This means that there exists a well determined probability per unit of time that the transformation by radioactive decay will take place. In this case, the probability is 0.5 per 6560 years, which is the half-life of ${ }^{240} \mathrm{Pu}$ nuclei. 
had the potentiality no longer exists; and thus, the potentiality does not exist at the same time as its actualization, i.e. the decay and its products.

The second condition of appropriateness that distinguishes the uses of the words "dispositionality" and "potentiality" is that it seems more natural to speak of potentiality if the actualization is not necessary but only possible (and probable with a probability $<1$ ). This second condition is closely linked to the first. It seems inappropriate to speak of a potentiality $\mathrm{P}$ for an actualization $\mathrm{A}$ if it is necessary that the object becomes a $\mathrm{B}$. The link with the first condition is this. Let us take it for granted that the system to which potentiality $\mathrm{P}$ is attributed is not isolated, which means that it is not shielded from the influence of factors from outside the system. Then, given that, according to the first condition of appropriateness, the actualization of a potentiality normally takes time, it is possible that some factor external to the system intervenes and prevents actualization. In other words, potentiality does not make its actualization necessary but only possible and more or less probable. There is no such constraint on the use of the concept of disposition. A disposition may or may not necessitate its manifestation, i.e. make it necessary that its manifestation comes about.

If the attribution of potentiality is inappropriate if the actualization is necessary, how can it be appropriate to attribute potentiality to deterministic systems? This is possible if and to the extent that a deterministic system is not isolated. In that case, the future state of the system can be predicted only with a certain probability. Probability values lower than one result from the possibility of disturbing influences from outside the system. Take the system consisting of the Earth and a massive object, which is put in a vacuum near the surface of the Earth. Is it necessary that the massive object falls toward the Earth with an acceleration $\mathrm{a}=9.81 \mathrm{~m} / \mathrm{s}^{2}$, so that it has, after a second of free fall, a speed of $9.81 \mathrm{~m} / \mathrm{s}$ ? It depends on whether it is isolated. In realistic circumstances, a system consisting of the Earth and one massive object is never isolated. In that case, it is appropriate to say that at the time it begins to fall, it has the potential, or the potentiality, to acquire, one second later, a speed of $9.81 \mathrm{~m} / \mathrm{s}$. By contrast, to the extent that it seems necessary that the body has, one second after beginning to fall, the speed determined by the law of free fall, i.e. $9.81 \mathrm{~m} / \mathrm{s}$ (i.e. insofar as one is justified to abstract away from outside influences, such as friction with air, that can interfere with the actualization), it is more natural to speak of the disposition to fall with an acceleration of 9.81 $\mathrm{m} / \mathrm{s}$, than of the potential to fall with that acceleration. Speaking of potentiality seems appropriate to the extent to which the possibility of interference from outside the system is taken into account ${ }^{17}$.

The possibility of external interference is not the only way in which our second condition of appropriateness may be satisfied. It is also natural to attribute potentiality to systems that follow an intrinsically indeterministic law of evolution. In their case, the law makes the actualization of the potentiality after a finite time possible but not necessary. Radioactive decay follows a probabilistic law. Such a law determines the probability with which, e.g., a given nucleus of ${ }^{240} \mathrm{Pu}$ decays during a given interval of time $\Delta \mathrm{t}$. For no $\Delta \mathrm{t}$ is its decay necessary even if the nucleus is perfectly isolated from its surroundings. Radioactive decay satisfies both conditions of appropriateness for the attribution of the concept of potentiality: it takes some finite time for a nucleus to decay, and for any given interval of time, it is only possible and probable to a precise extent, but not necessary, that it will decay during that interval.

17 This presupposes that one does not take the experimenter into account as being herself part of the system. The experimenter can decide to let the object fall or not. Thus, if the experimenter is taken to be part of the system, it is perfectly appropriate to attribute to the body the potentiality to fall with $9.81 \mathrm{~m} / \mathrm{s}^{2}$, even if the system is otherwise isolated. But in that case the application of the notion of potentiality is not justified on physical grounds but because it is appropriate to attribute the experimenter the freedom of choice. Being not purely physical, such systems lie outside the scope of the present chapter. 
Here is a hypothesis about the ground of these intuitive differences in the appropriateness of the use of the terms "potentiality" and "disposition". They are due to the fact that the concept of potentiality has its origin in the Aristotelian concept dynamis. Aristotle uses the concepts of potentiality/actuality (dynamis/energeia or, in the latin translation of Aristotle's greek terms, potentia/actus) to explain change in general, all across nature. Biological applications of Aristotle's distinction between dynamis and energeia are paradigmatic: Seeds have the potential of growing into corn (Metaphysics, $\Theta$ 8, 1049b20). The actual growing of the corn can be understood as a gradual transition from dynamis to energeia, or, in the usual translation, from potentiality to actuality. The grown corn possesses in actuality the form that the seed possesses only potentially. It is essential for this "transition from potentiality to actuality" that it takes time, the exact amount of time being determined both by the nature (form) of the potentiality and the circumstances, which include such factors as abundance of nutrients and water. There is no definite and unique instant at which the seed ceases to be corn only potentially and becomes an actual corn plant. Be that as it may, the growth of a biological organism cannot be instantaneous. It is a paradigmatic case of potentiality, in that the transition from potentiality to actuality follows an evolution necessarily extended in time and structured by a succession of intermediate stages. In the analysis of processes of change, potentiality characterizes the earlier stages, whereas actuality characterizes later stages ${ }^{18}$. This explains why it is more natural to speak of potentiality in cases in which actualization takes time.

\section{Potentiality in quantum mechanics.}

\subsection{Heisenberg's interpretation of quantum mechanical systems in superposed states}

In the Gifford lectures that Werner Heisenberg delivered in St Andrews in the winter of 1955/56, he suggested to interpret the quantum mechanical wave function as describing a "strange kind of physical reality", which he characterised as being "just in the middle between possibility and reality" (Heisenberg 1958, 15). On the same occasion, he suggested interpreting this new kind of reality as "a quantitative version of the old concept of 'potentia' in Aristotelian philosophy" (1958, 15). According to Heisenberg's version of the so-called Copenhagen interpretation ${ }^{19}$, certain quantum mechanical systems - those that are in so-called "superposed states" - are in an objectively undetermined state as long as they are not observed, i.e. as long as they have not come into interaction with a measurement apparatus. It is essential to the Copenhagen interpretation that the measurement apparatus is described in the framework of classical physics. The apparatus itself is not in any indeterminate quantum state. At the moment of measurement, the quantum system, which has up to that point been in a state of "potentiality" ("potentia", p. 15, 154, 155), "possibility" (e.g. p. 21, 24, 28) or "tendency" (e.g. p. 15, 24, 28, 155) - which are all terms Heisenberg uses interchangeably undergoes a sudden change and enters into a determinate actual state, so that it becomes a part of the same kind of reality as the measurement apparatus, which can be described in the terms of classical physics.

There are at least three ways of interpreting Heisenberg's claim that the event of measuring an observable of a quantum system makes the system undergo a "transition from

\footnotetext{
18 This is compatible with Aristotle's thesis (Metaphysics, $\Theta$ 8, 1049b20) that actuality also precedes potentiality, in the sense that seeds can only be produced by actual grown corn plants.

Howard (2004) shows that the Copenhagen interpretation of quantum mechanics has been created by Heisenberg in the mid-1950s, and that Heisenberg's presentation of this doctrine differs from Bohr's interpretation of quantum mechanics, with which is it often identified. Cf. Faye (2008).
} 
the possible to the actual" (this expression occurs frequently in Heisenberg (1958), in particular in chapters 3 and 8, e.g. p. 28, p. 116).

On the first interpretation (1) of this claim, a quantum mechanical system that is in a superposed state has, before measurement, only potential existence. It only becomes actual, or real, upon measurement. On a different interpretation (which we will further subdivide in interpretations (2) and (3)), the system itself is actual or real even before the measurement. However, it has some of its properties only potentially, i.e. properties that correspond to physical observables, with respect to which the system is not in any eigenstate but rather in a linear superposition of eigenstates.

Suppose F is an "observable", i.e. an operation of measurement (mathematically represented by an operator), such as spin in a given direction, which has only two eigenvalues $\mathrm{f}_{1}$ and $\mathrm{f}_{2} \cdot{ }^{20}$ Let $\mathrm{u}_{1}$ and $\mathrm{u}_{2}$ be the eigenstates of a quantum mechanical system corresponding to these eigenvalues, such that if the system is in eigenstate $u_{1}$, a measurement of $F$ will yield the result $\mathrm{f}_{1}$, and if it is in eigenstate $\mathrm{u}_{2}$, a measurement of $\mathrm{F}$ will yield result $\mathrm{f}_{2}$. According to the superposition principle, the system can also be in a superposed state $\mathrm{u}$, which can be represented as a linear combination of eigenstates: $u=a_{1}+b_{2}$, where $a^{2}+b^{2}=1$. Such a superposed state cannot be directly observed. What can be confirmed by observation is that the probability that the measurement of $F$ delivers the eigenvalue $f_{1}$ is $a^{2}$ and the probability that it delivers the eigenvalue $f_{2}$ is $b^{2}$.

A relatively simple experimental situation of this sort is a stream of silver atoms, which are prepared by a Stern-Gerlach apparatus so as to be all in the same determinate state of their spin. Let this apparatus, called a "polarizer", be so oriented that all atoms exit it in state spin-up $\left|\sigma_{z}^{+}\right\rangle$in the direction of the z-axis. Then, with respect to a measurement of spin in a different direction $\mathrm{v}$, which makes an angle $\theta$ with the $\mathrm{z}$-axis, these atoms are all in the superposed state

$$
u=\cos \frac{\vartheta}{2}\left|\sigma_{v}^{+}\right\rangle-\sin \frac{\vartheta}{2}\left|\sigma_{v}^{-}\right\rangle,
$$

where $\left|\sigma_{v}^{+}\right\rangle$and $\left|\sigma_{v}^{-}\right\rangle$are the eigenstates of the system with respect to a measurement of spin in direction $v$. When a second Stern-Gerlach apparatus, used as an analyzer, measures the spin of these atoms in direction $v$, the probability that the measurement yields the result spin-up is $\cos ^{2} \frac{\vartheta}{2}$

and the probability that the measurement yields the result spin-down is $\sin ^{2} \frac{\vartheta}{2}$.

The second and third interpretations of Heisenberg's claim mentioned above both take the system itself to be actual before measurement but to be only potentially in a certain state. According to interpretation (2), the system's state with respect to observable F before measurement is actual, although it is not an eigenstate but a linear superposition of eigenstates of spin in v-direction. What is potential about the system before measurement are the possible results of measurement, i.e. the eigenvalues $f_{1}$ and $f_{2}$, and the eigenstates in which the system ends up after measurement of $\mathrm{F}$.

According to interpretation (3), the system itself is actual before measurement but it is not in any determinate actual state with respect to observable F. In other words, the superposed state itself is only a potential and not an actual state. Before measurement of $\mathrm{F}$, the

\footnotetext{
${ }^{20}$ In a realistic interpretation, each observable corresponds to a property. However, I will follow the standard way of introducing observables in terms of mathematical operations on representations of physical systems.
} 
system is in an indeterminate state, and indeterminate states cannot be actual. Therefore, only a measurement of $F$ puts the system in an actual state with respect to $F$.

Some passages suggest that Heisenberg intends the first interpretation (1). "The ontology of materialism" he says, "rested upon the illusion that the kind of existence, the direct 'actuality' of the world around us, can be extrapolated into the atomic range. This extrapolation is impossible, however" (1958, p. 119). This suggests that only the macroscopic objects and phenomena accessible to direct observation are "actual", whereas everything belonging to the "atomic range", i.e. quantum mechanical systems, are not actual and exist only potentially.

However, this interpretation runs into the following difficulty. Heisenberg's claim according to which a quantum system undergoes, at the moment of measurement, a transition from possibility to actuality, cannot mean that, when a system is measured, it goes from a state of possible existence into a state of real existence, simply because at a time at which it only has possible existence, it has no existence at all, and a non-existent system, quantum mechanical or not, cannot enter into any interactions and cannot in particular undergo any measurement. So no measurement could bring "it" into actual existence if it had not been actual before the measurement.

In other passages, Heisenberg suggests that what is intended is the second interpretation (2). "The 'reduction of wave packets' always appears in the Copenhagen interpretation when the transition is completed from the possible to the actual. The probability function, which covered a wide range of possibilities, is suddenly reduced to a much narrower range by the fact that the experiment has led to a definite result, that actually a certain event has happened." (Heisenberg 1958, p. 116).

In the interpretation suggested in this passage, what is potential but not actual is neither the very existence of the system that is in a superposed state with respect to an observable $\mathrm{F}$ nor its superposed state with respect to variable F. What is potential and not actual are only the different results of measuring $F$, i.e. the eigenvalues of $F$. In this interpretation, measurement makes the system change, from an actual superposed state to an actual eigenstate. What is potential is only the possession of one of the eigenstates before the system is measured.

According to the third interpretation (3), the system before measurement actually exists but it is only potentially in a superposed state with respect to observable $\mathrm{F}$. This raises two difficulties. The first is that it makes it difficult to justify the existence of the system, for the same reasoning seems to apply to all its observables, and it is not clear how the system could itself actually exist while its states with respect to observables all exist only potentially. This is just the difficulty faced by interpretation (1). The second difficulty is that no single measurement could make this potential state actual for no single measurement can yield the result that the system is in a superposed state. Measurements of $\mathrm{F}$ can yield only eigenvalues. Once measured, the system enters and remains in one of the available eigenstates, so that repeated measurements will keep yielding the same eigenvalue.

One way to justify the first (1) interpretation of Heisenberg's thesis that quantum systems in superposed states exist only potentially but not actually before they have interacted with a measurement apparatus (or the third (3) interpretation according to which they are, before a given observable is measured, in a potential state with respect to that observable), is the neo-Kantian interpretation of quantum mechanics. In this interpretation, objective knowledge requires the application of categories to the sensory input that reach the sensory organs of the knower, coming from hypothetical things in themselves. By the very nature of knowledge as the result of the structuring of the brute sensory input by categories, any known object must be structured by these categories. From this perspective, a quantum system cannot be known as it is in itself because it provides only part of the input that leads to objective 
knowledge, the rest being provided by the measurement apparatus, which plays the role of the Kantian subject of experience and knowledge ${ }^{21}$. This may be taken as a reason to claim that either the system itself (interpretation 1) or the states it is in, such as the superposed state sketched above (interpretation 3 ), has only potential existence.

However, this approach does not appear to be helpful for making sense of the measurement of quantum mechanical systems in superposed states. Either the measuring event is interpreted as requiring a subject of experience, i.e. an observer who imposes structure on the quantum system to know its properties. In this case the quantum system, as it is "in itself" before measurement remains unknown and unknowable; it remains therefore impossible to understand how it evolves to become part of ordinary reality. Or measurement does not require a human subject of experience because a mechanical measuring device can play the role of the subject imposing on the quantum system the structure of its categories. The problem is the same: the main thesis of the neo-Kantian approach in both versions (measuring requiring a subject of experience or only an apparatus) is that the quantum system before measurement is unknowable, because it has not yet undergone the process of objectification by being structured by categories. If it is impossible in principle to know the quantum mechanical system before measurement, it is also impossible to understand what happens when such an unknowable system undergoes a measurement. Maybe one might call it a "skeptical" theory of the nature of quantum systems; it might rather be a way of abandoning the search for a theory.

\subsection{A realist interpretation of quantum mechanical systems in superposed states, in terms of powers and dispositions}

The concept of potentiality can be used to understand quantum systems in a superposed state before measurement, without abandoning a realist interpretation of the quantum mechanical description of such systems, both with respect to the system itself and with respect to the superposed state. In other words, the concept of potentiality makes it possible to stick to the thesis that both the system and its state with respect to observable $\mathrm{F}$ exist independently of their observation or measurement, and to avoid the sceptical conclusion that the system before measurement is unknowable. According to the second interpretation (2) of Heisenberg's claim that measurement induces a transition from potentiality to actuality, what undergoes such a transition is neither the system itself, with respect to its very existence, nor the superposed state, but only one of the eigenstates that are part of the superposition. If the system is in a superposition of eigenstates $u_{1}$ and $u_{2}$, then the system is only potentially in each eigenstate. However, it does not follow that it is only potentially in a superposed state (interpretation 3 ), or that it exists only potentially before measurement (interpretation 1 ). One must not confuse interpretations (2) and (3): What does it mean to say that the system (which actually exists) has the potential to evolve into eigenstate $u_{1}$, as well as the potential to evolve into one eigenstate $u_{2}$ ? It means that the system is potentially, and not actually, in eigenstate $\mathrm{u}_{1}$; and that it is potentially, but not actually, in eigenstate $\mathrm{u}_{2}$. It does not mean that the property of being potentially in each of the eigenstates is itself only potential and not actual. After all, it is the property of being in a superposed state that causes, together with the interaction of measurement, the result of measurement. If the property of being in a superposed state is causally efficacious it must be real.

Antirealist interpretations of quantum systems in superposed states are motivated by the question: Which value of the observable $\mathrm{F}$ does the system have before $\mathrm{F}$ is measured? It

\footnotetext{
$21 \quad$ I am well aware that this sketch of a neo-Kantian interpretation of quantum system is more a caricature than a faithful presentation. Neo-Kantian interpretations of quantum mechanics have been worked out by d'Espagnat (1994) and Bitbol (1996).
} 
seems unavoidable to answer that question by saying that the system has "no definite value at all" (Shimony 1978, p. 4). Another way of expressing this idea is to say that the system is in an "indefinite" state. If the system is in a "situation of indefiniteness", it seems to follow that it isn't in any real state at all, or constitues, in Heisenberg's words, "a strange kind of physical reality just in the middle between possibility and reality" (Heisenberg 1958, p. 15). But this conclusion can be avoided. To be sure, before measurement of an observable with respect to which it is in a superposed state, it has no definite manifest properties with respect to that observable, i.e. it is not in any of the eigenstates corresponding to the observable. But the "indefiniteness of dynamical variables" (Shimony 1978, p. 5) does not extend beyond the eigenstates. It is compatible with the hypothesis that a system in a superposed state is in a definite state with respect to a non-manifest theoretical property, which we know only in a dispositional way: a power, or powerful property.

The metaphysical interpretation of quantum mechanical state descriptions in such dispositional terms has been explored by a number of authors ${ }^{22}$. Popper (1957) was the first to suggest a realist interpretation of the potentiality that the quantum mechanical state description attributes to quantum systems in superposed states ${ }^{23}$. Superposed states cannot be directly observed. Their attribution to a system is subject to the usual methodology of theoretical terms: It is reasonable to attribute a system a theoretical, i.e. not directly observable property $\mathrm{T}$ if and only if the hypothesis that the system possesses $\mathrm{T}$ provides the best available explanation of its observable behaviour ${ }^{24}$. Superposed states are theoretical states. However, this is no reason to interpret them as something that exists only potentially but not actually. True, the system (in the superposed state) cannot also be actually in one of the eigenstates. Can the system both be potentially in state $\mathrm{u}_{1}$ and be potentially in state $\mathrm{u}_{2}$ ? As eigenstates, states $u_{1}$ and $u_{2}$ are orthogonal, which means that they are incompatible. Thus it may seem contradictory to suppose that the system may be in some sense in both states at the same time.

However, the contradiction is only apparent. Indeed, the concepts of potentiality and actuality, or equivalently, of power, disposition and manifestation, allow us to construct a realist interpretation of the superposed state. This is possible by applying to the case of superposed quantum states a metaphysical account that is also required to account for ordinary so-called "multitrack" powers (Kistler 2012). It will become clear in a moment why it is preferable to speak of multitrack powers rather than dispositions. A dispositional concept is called "multitrack" if its meaning is linked to several, not only one, counterfactual conditionals. A concept is called single-track if its meaning associates it to just one counterfactual conditional, linking it to a specific manifestation in one testing condition. However, all scientific concepts that figure in different laws are multi-track. Take electric charge. Figuring in many laws, electric charge q gives any charged object many dispositions:

\footnotetext{
22 The accounts that have been developed in a realist framework differ considerably, and I cannot here do justice to any of them in detail. See, e.g., Popper (1957), Suppes (1974), Shimony (1978), Sfendoni-Mentzou (1993), Sfendoni-Mentzou (1994), Dorato (2007), Koznjak (2007), Karakostas (2007), Suarez (2007).

However, Popper interprets propensities, which correspond to the potential properties we are considering, as "relational properties of the experimental set-up" $(1957,68)$. The propensity of a fair die to land on 6 with a probability of $1 / 6$ is a relational property that the die has relative to the set-up of throwing dice by hand on a flat surface. Dorato (2007) follows Popper's lead in taking the dispositionality of quantum states as a consequence of their relationality. Taking up a distinction introduced by Clifton and Pagonis (1995), Dorato argues that a superposed quantum state is not only "contextual," (which means that the measured value is possessed by the system before measurement and only revealed by the measurement) but "contextual 2 ", which means that the measured value is in part determined by the interaction with the measuring apparatus.

$24 \quad$ What exactly it means to be a good explanation has been the subject of much work in the philosophy of science. The most important criteria for what makes for a good explanation are strength (the range of observable facts it can explain), simplicity (the number and simplicity of the assumptions it makes), and fruitfulness (the number and variety of new, not yet observed phenomena it suggests).
} 
1. the disposition to attract a second object with charge $\mathrm{q}^{*}$ with $F=k_{e} \frac{q \cdot q^{*}}{r^{2}}$ (Coulomb force), where $\mathrm{k}_{\mathrm{e}}$ is Coulomb's constant and $\mathrm{r}$ is the distance between the objects.

2. The disposition to undergo a force $\mathbf{F}=\mathrm{q} \mathbf{E}$ if it were in $\mathbf{E}$, where $\mathbf{E}$ is the electric field.

3. The disposition to undergo $\mathbf{F}=q \mathbf{v} \times \mathbf{B}$ (Lorentz force) if it were moving with $\mathbf{v}$ in $\mathbf{B}$, where $\mathbf{v}$ is the object's speed and $\mathbf{B}$ the magnetic field.

4. The disposition to create magnetic moment $\mathbf{m}=1 / 2 \mathrm{q} \mathbf{r} \times \mathbf{v}$ if it were in rotation with speed $\mathbf{v}$ on radius $\mathbf{r}$.

The property of being electrically charged is a theoretical property that underlies a whole set of dispositions. Let me explain this idea. The content of a theoretical property is exhaustively specified by the content of the laws containing the property. These laws indicate how objects having the property evolve. One can express this by saying that each law containing a theoretical property expresses a disposition of objects having the property. It is the fact that a given object is electrically charged that makes true the attribution of all the dispositions in this set to the object. I suggest calling this property, which contributes to make true the attribution of all the different dispositions in this set, the power, or powerful property, underlying the set of dispositions. Every disposition has a causal basis: The causal basis of the disposition consists of the conjunction of intrinsic properties of the object possessing the disposition, which contribute causally to the manifestation of the disposition. A given set $\left\{D_{1}, \ldots, D_{n}\right\}$ of dispositions $D_{i}$ characterizes a unique underlying power if and only if it satisfies the following criterion: If there is a set of intrinsic properties $\left\{\mathrm{P}_{1}, \ldots, \mathrm{P}_{\mathrm{n}}\right\}$, such that all properties $P_{i}$ in this set belong to the causal basis of each disposition $D_{i}$, then there is a single powerful property underlying the whole set of dispositions, namely the conjunction of these Pi's. This power, or powerful property, is what has traditionally been called a multitrack disposition.

This framework can be used to construct a realist interpretation of superposed states of quantum mechanical systems. A superposed state can be seen as a multitrack powerful state. This means that the fact that a system is in that state makes it the case that it has a set of dispositions or potentialities. Let us once again consider our stream of silver atoms, which have been prepared to be in a unique state with respect to their spin, which is oriented along the positive z-axis. Being in that state gives the system different dispositions, one for each angle at which their spin can be measured by a Stern-Gerlach analyzer. Each measurement at a given angle $\theta$ with respect to the $\mathrm{z}$-axis can be seen as a triggering condition. There is an important aspect by which the spin state of this quantum system differs from an ordinary powerful property. A property such as electric charge, which we have mentioned above, gives a physical system various dispositions, where each disposition corresponds to a unique manifestation. For quantum systems such as silver atoms with spin, the manifestations for a given triggering condition are in general only probabilistically determined. In other words, for a given triggering condition, several manifestations are possible, only the probability of which is determined by the state of the system and the triggering condition. For a general direction $\mathrm{v}$ of measurement that makes an angle $\theta$ with the z-axis, the system is, with respect to the observable spin along the v-axis, in a superposed state $u=\cos \frac{\vartheta}{2}\left|\sigma_{v}^{+}\right\rangle-\sin \frac{\vartheta}{2}\left|\sigma_{v}^{-}\right\rangle$, where $\left|\sigma_{v}^{+}\right\rangle$and $\left|\sigma_{v}^{-}\right\rangle$are the eigenstates of the system with respect to the measurement of spin along direction $\mathrm{v}$. If the spin is measured along the $\mathrm{v}$-axis, the manifestations are only probabilistically determined. For such a mesurement, the probability that a given atom will be 
found in state spin-up is $\cos ^{2} \frac{\vartheta}{2}$, whereas the probability that it will be found in a state spindown is $\sin ^{2} \frac{\vartheta}{2}$.

The dispositions to manifest spin in arbitrary directions are all probabilistic except for the direction of the z-axis, relative to which the atoms are in an eigenstate with respect to their spin, after their interaction with the polarizer.

This description of the situation corresponds to our interpretation (2) of Heisenberg's claim that a system in a superposed state is, before it is measured, in a state of potentiality. Unlike interpretation (1) of that claim, the system before measurement actually exists. Unlike interpretation (3), the system possesses an actual powerful state. This powerful state, which can be represented as a linear combination of eigenvectors, makes true the attribution of a set of dispositions to the system for specific results of measurement, with specific probabilities. What is potential, in the system before measurement, are the manifestations of each disposition, i.e. the measurement results in each direction. For each direction $\mathrm{v}$ of measurement, the two possible eigenstates of spin according to the $\mathrm{v}$ direction are, before measurement, only potential and not actual.

Here it may be useful to add a remark about the distinction between the dispositional and the categorical. It may seem paradoxical to hypothesize that the superposed state of a quantum system is an actual property although it can be characterized only in terms of counterfactual conditionals. Being dispositional seems to be incompatible with being actual if it is presupposed that (1) only categorical properties can be actual ${ }^{25}$ and (2) no property can be both categorical and dispositional.

Presuppositions (1) and (2) lie behind an influential argument due to Armstrong (1997) according to which it is impossible that all fundamental physical properties are dispositional $^{26}$. This would imply, according to Armstrong, that physical properties are only potentialities, interpreted as non-actual possibilities. Here is Armstrong's argument. If all properties are dispositional, their manifestations are also dispositional, because they are themselves properties. Armstrong presupposes 1) that dispositional properties are potentialities, or as he says "potencies", which have only potential but not actual existence, which implies that 2) a dispositional property cannot also be categorical because categorical properties are actual. Now, he argues, if the manifestation of a disposition is only another disposition, then nothing ever gets actualized and the world remains in a state of potentiality, which is absurd. "Can it be that everything is potency, and act is the mere shifting around of potencies? ... Given a purely Dispositionalist account of properties, particulars would seem to be always re-packing their bags as they change their properties, yet never take a journey from potency to act. For 'act' on this view, is no more than a different potency." (Armstrong 1997, p. 80).

The absurd result that there are no actual properties at all can be avoided by taking the dispositional-categorical distinction to be a semantic distinction between predicates, rather than as a metaphysical distinction between properties. In this sense, a predicate is dispositional if and only if its meaning implies analytically (i.e. merely by virtue of its meaning) that certain counterfactual conditionals are true of the objects to which the predicate applies. ' $\mathrm{X}$ is water-soluble' implies analytically that, if $\mathrm{x}$ were put into water, $\mathrm{x}$ would dissolve. Attributions of categorical predicates also imply such counterfactual conditionals,

\footnotetext{
${ }^{25}$ According to this presupposition, which I take to be unjustified and mistaken, dispositions are non-actual potentialities.

$26 \quad$ Another version of the argument can be found in Armstrong $(2000,14)$.
} 
but in their case the implication is a posteriori: its truth is not guaranteed by the mere meaning of the predicate, but rather by laws (known only a posteriori) applying to the property ${ }^{27}$.

If the distinction between the categorical and the dispositional is conceptual and semantic rather than metaphysical, it is possible to conceive of the same property both in a dispositional and in a categorical way. When one ascribes the property by stating how it would manifest in certain triggering circumstances, it is conceived in a dispositional way. However, this is compatible with the possibility of referring to the same property in a categorical way, by stating how it is independently of its manifestations in various circumstances. True, categorical descriptions are generally more difficult to find than dispositional descriptions. What is electrical conductivity in itself apart from being the property of being such that, if field $\mathrm{E}$ is applied, current with density $\mathrm{J}=\sigma \mathrm{E}$ flows? However, the fact that we can describe the property only in a dispositional way is no reason to doubt its reality or causal efficacy.

Superposed states of quantum mechanical systems are states that we can describe only in a dispositional way. To describe such a state as a superposition or linear combination of eigenstates of a certain observable, is to describe it in a dispositional way: If a measurement of the observable was carried out, the various available results would manifest with frequencies given by the squares of the linear factors of the superposition. However, by parity of reasoning with the case of conductivity, the fact that we can describe the superposed state only dispositionally does neither entail that the state is not actual (i.e. that the system has the state only potentially as in interpretation 3 above) nor that it is in principle impossible to conceive it in a categorical way.

With respect to the question of the reality of quantum states, it is important to avoid the mistake of interpreting potentiality as possibility, which comes to conflating interpretations 2 and 3 . If a system has at time $t$ the potential to acquire property $\mathrm{P}^{*}$ at $\mathrm{t}^{*}$, then it is true that it is, at time $t$, a mere possibility that it will have $\mathrm{P}^{*}$ at time $\mathrm{t}^{*}$. This corresponds with interpretation 2. However, it would be a mistake to conclude from this (with interpretation 3 ) that the possession, at time $t$, of the potentiality is itself a mere possibility. On the contrary, the potentiality is a real property (it is a power, or powerful property), and as such it is capable of contributing causally to the system's acquisition of property $\mathrm{P} *$.

\section{Conclusion}

The term "potentiality" is nowadays used neither in physics nor in most philosophical interpretations of physical theories. Nevertheless, I have argued that it can be helpful to use it in the context of the metaphysical interpretation of both classical and quantum physics. Potentiality is only pragmatically different from dispositionality. In other words, something

27 This conception is defended by Shoemaker (1980), Mumford (1998), Mellor (2000), Gnassounou and Kistler (2007), Mumford (2007), Kistler (2007). Bird (2007, 100-4) develops a similar argument to the one I sketch here against Armstrong's claim that dispositional properties have "too little actuality". There is another way of reconciling the fact that most properties can be conceived in a dispositional way with the fact that they can also be actual. According to an account due to Martin (1996) and Heil (1998), each property has a "dual nature" (Heil 1998, 182), in the sense that it "endows its possessor with both a particular disposition or "causal power' and a particular quality" (Heil 1998, 181). However, rather than solving the problem of understanding how these two apparently incompatible "aspects" can coexist, this might just be a way of stating it. As a metaphysical account of the nature of properties, Martin's and Heil's account also faces the following difficulty that is avoided by the semantic account. If every property has a dual nature, then every property is dispositional (cf. Popper 1957). But then, “dispositional”, taken as a metaphysical concept, doesn't satisfy a necessary condition of concepts: to partition the universe of discourse in objects falling into its extension and objects not falling in it. 
has potentiality if and only if it has dispositionality. However, it can be more or less appropriate to speak of potentiality rather than of dispositionality. I have suggested that the use of potentiality is appropriate to the extent that two conditions are satisfied: 1) the actualization takes (more or less) time and 2) the potentiality makes its actualization (more or less) probable but not necessary. The use of "potentiality" is the more appropriate the longer the actualization takes and the less it is necessitated by the potentiality. The use of "dispositionality" is not subject to such restrictions. In classical physics, physical potentials (such as the gravitational potential) and capacities (such as heat capacity) are cases of disposition and potentiality. Many other properties, such as electric charge, are dispositional because they can be defined in a dispositional way, by various counterfactuals linking triggering conditions to manifestations. It is a consequence of the pragmatic difference between the conditions of appropriate use of the terms "potential" and "dispositional" that it is less appropriate to attribute an object the potential for property $\mathrm{P}$ at a time at which $\mathrm{P}$ is already actualized, whereas there is no pragmatic obstacle to the attribution of a disposition to an object at a time at which the disposition is manifested.

Heisenberg used the concept of potentiality for the metaphysical interpretation of quantum mechanical systems in superposed states. After having shown that Heisenberg's thesis leaves room for several interpretations, I have taken up one of them and sketched a realist interpretation of quantum systems in superposed states that uses the concepts of power and disposition. Here are the main theses of this interpretation. 1) A quantum system that is, with respect to a given observable, in a superposed state, is potentially (but not actually) in one of its available eigenstates. 2) The superposed state itself is a powerful state that plays the role of a theoretical property: It provides a unifying explanation of a set of dispositions, corresponding to the measurement of various observables, where the measurement is the triggering condition and the outcome the manifestation. ${ }^{28}$

\section{References}

Armstrong, D. M. (1973), „Beliefs as States”, in: Raimo Tuomela (ed.), Dispositions, Dordrecht: Reidel 1978, 411-425.

Armstrong, D. M. (1997), A World of States of Affairs, Cambridge: Cambridge University Press.

Armstrong, D.M. (2000), "The Causal Theory of Properties: Properties according to Shoemaker, Ellis and others", Metaphysica 1, 5-20.

Belot, G. (1998), "Understanding electromagnetism", British Journal for the Philosophy of Science, 49 (4), 531-555.

Bird, A. (1998), "Dispositions and antidotes", Philosophical Quarterly 48, 227-234.

Bird, A. (2007), Nature's Metaphysics. Laws and Properties, Oxford: Clarendon Press.

Bitbol, M. (1996), Mécanique quantique, une introduction philosophique, Paris: Flammarion. Blackburn, S. (1990), "Filling in Space", Analysis 50, 62-65.

Cartwright, N. (1989), Nature's Capacities and their Measurement, Oxford: Oxford University Press.

Clifton R. and Pagonis C. (1995), "Unremarkable Contextualism: Dispositions in the Bohm theory", Foundations of Physics 25, 2, 281-296.

28 Work for this chapter was funded by ANR research project ANR-12-BSH3-0009. I thank K. Engelhard, A. Guay, S. Le Bihan, P.-M. Morel and M. Schüle for very helpful feedback and criticisms on earlier versions of this chapter, 
D’Espagnat, B. (1994), Le Réel voilé, analyse des concepts quantiques, Paris: Fayard; transl. Veiled Reality: An Analysis of Present-Day Quantum Mechanical Concepts, Boulder/Colorado: Westview Press.

Dorato, M. (2007), „Dispositions, Relational Properties, and the Quantum World”, in: M. Kistler and B. Gnassounou (2007), 249-70.

Faye, J. (2008), "Copenhagen Interpretation of Quantum Mechanics", Stanford Encyclopedia of Philosophy, http://plato.stanford.edu/entries/qm-copenhagen/

Feynman, R.P., Leighton R.B., Sands M. (1963), The Feynman Lectures on Physics. Vol. 1. Mainly Mechanics, Radiation, and Heat. Reading, Massachusetts: Addison-Wesley, pages?.

Feynman, R.P., Leighton R.B., Sands M. (1964), The Feynman Lectures on Physics. Vol 2. Mainly Electromagnetism and Matter. Reading, Massachusetts: Addison-Wesley, pages?.

Gnassounou, B. and Kistler, M. (2007), „Introduction”, in: M. Kistler and B. Gnassounou (2007), 1-40.

Healey, R. (1997), "Nonlocality and the Aharonov-Bohm effect", Philosophy of Science 64, $18-41$.

Heil, J. (1998), Philosophy of Mind. A Contemporary Introduction, London: Routledge.

Heisenberg, W. (1958), Physics and Philosophy. The Revolution in Modern Science. New York: Harper Collins.

Howard, D. (2004), "Who Invented the 'Copenhagen Interpretation?' A Study in Mythology", Philosophy of Science 71, 669-682.

Karakostas, V. (2007), "Nonseparability, Potentiality, and the Context-Dependence of Quantum Objects", Journal for General Philosophy of Science 38, 279-97.

Kistler, M. (2007), „The Causal Efficacy of Macroscopic Dispositional Properties”, in: M. Kistler and B. Gnassounou (2007), 103-32.

Kistler M. (2012), „Powerful properties and the causal basis of dispositions”, in A. Bird, B. Ellis, and H. Sankey (eds.), Properties, Powers and Structures. Issues in the Metaphysics of Realism, New York, Oxford: Routledge, 119-137.

M. Kistler and B. Gnassounou (eds.) (2007), Dispositions and Causal Powers, Aldershot: Ashgate.

Koznjak, B. (2007), "Möglichkeit, Wirklichkeit und Quantenmechanik", Prolegomena 6/2, 223-252.

Lewis, D. (1997). “Finkish Dispositions", Philosophical Quarterly 47, 143-158.

Martin, C. B. (1996), „Final Replies to Place and Armstrong”, in : Armstrong, David M., C.B. Martin et U.T. Place, Dispositions: A Debate, Tim Crane (ed.), London: Routledge, 163-192.

McKitrick, J. (2003), “The Bare Metaphysical Possibility of Bare Dispositions", Philosophy and Phenomenological Research 64, 349-369.

Mellor, D.H. (2000), "The Semantics and Ontology of Dispositions", Mind 109, 757-780.

Molnar, G.(2003), Dispositions. A Study in Metaphysics. Oxford: Oxford University Press.

Mumford, S. (1998), Dispositions, Oxford: Oxford University Press.

Mumford, S. (2006), “The Ungrounded Argument", Synthese 149, 471-489.

Mumford, S. (2007), „Filled In Space”, in: M. Kistler and B. Gnassounou (2007), 67-80.

Mumford, S. and Anjum, R.L. (2011), Getting Causes from Powers, Oxford: Oxford University Press.

Popper, K. (1957), „The Propensity Interpretation of the Calculus of Probability, and the Quantum Theory”, in S. Körner (ed.), Observation and Interpretation, London: Butterworth, 65-70.

Prior E. W., Pargetter R., Jackson F. (1982), "Three Theses about Dispositions", American Philosophical Quarterly 19, 251-257. 
Quine W. V. (1966), „Necessary Truth”, in: The Ways of Paradox and Other Essays, Cambridge (Mass.): Harvard University Press, 68-76.

Quine, W.V.O. (1971), The Roots of Reference, LaSalle, Ill.: Open Court.

Sfendoni-Mentzou, D. (1993), „The Role of Potentiality in Pierce's Tychism and in Contemporary Discussions in Quantum Mechanics and Microphysics”, in: E.C. Moore (ed.), Charles S. Peirce and the Philosophy of Science, Tuscaloosa, London: The University of Alabama Press, 246-261.

Sfendoni-Mentzou, D. (1994), "Laws of nature: ante res or in rebus?", International Studies in the Philosophy of Science 8/3, 229-242.

Shimony, A. (1978), "Metaphysical Problems in the Foundations of Quantum Mechanics", International Philosophical Quarterly 8, 3-17.

Shoemaker, S. (1980), "Causality and Properties", repr. in S. Shoemaker, Identity, Cause and Mind, Cambridge: Cambridge University Press, 206-233.

Suarez, M. (2007), Quantum propensities, Studies in History and Philosophy of Modern Physics 38, 418-438.

Suppes, P. (1974), “Aristotle's Concept of Matter and its Relation to Modern Concepts of Matter", Synthese 28, 27-50. 